\title{
A Rare Cause of Endocarditis: Streptococcus pyogenes
}

\author{
Ayşegül Yeşilkaya1, Özlem Kurt Azap ${ }^{1}$, Bahar Pirat², Bahadır Gültekin ${ }^{3}$, Hande Arslan ${ }^{1}$ \\ 'Department of Infectious Diseases and Clinical Microbiology, Faculty of Medicine, Başkent University, Ankara, Turkey \\ ${ }^{2}$ Department of Cardiology, Faculty of Medicine, Başkent University, Ankara, Turkey \\ ${ }^{3}$ Department of Cardiovascular Surgery, Faculty of Medicine, Başkent University, Ankara, Turkey
}

\begin{abstract}
Although group A $\beta$-hemolytic streptococcus is an uncommon cause of infective endocarditis, an increase in the incidence of invasive group A streptococcus infections including bacteremia has been reported in the last two decades. Herein we report Streptococcus pyogenes endocarditis in a previously healthy adult patient who was hospitalized to investigate the etiology of fever. Because of a suspicion of a new vegetation appeared in the second (aortic) valve in the $14^{\text {th }}$ day of high dose penicillin $\mathrm{G}$ treatment, the mitral and aortic valves were replaced by mechanical prosthesis on the $22^{\text {nd }}$ day of treatment. He was discharged from hospital after the 6 week course of antibiotic treatment.
\end{abstract}

Key Words: Group A $\beta$-hemolytic streptococcus, endocarditis

\section{Introduction}

The microbiology of infective endocarditis (IE) has changed in the last decade (1). IE due to group A $\beta$-hemolytic streptococcus (Streptococcus pyogenes) has rarely been reported in the literature $(2,3)$. We herein report $S$. pyogenes native mitral and aortic valve endocarditis in an elderly patient who had been hospitalized with fever.

\section{Case Report}

A 71-year-old male was admitted to our department with a 4-day history of chills and fever despite oral ciprofloxacin treatment which was given for the presumptive diagnosis of urinary tract infection. Recent dental treatment, recent surgery and drug abuse was absent. His past cardiac history was unremarkable except for hypertension. Abnormal findings on physical examination were pyrexia of $39.8^{\circ} \mathrm{C}$, hyperemia of pharynx and tachycardia. Abnormal laboratory findings were high C-reactive protein $(74.5 \mathrm{mg} / \mathrm{L})$ and 4 red cells per high power field in urinalysis. A couple of blood cultures and urine culture were drawn after admission for the evaluation of fever etiology. Levofloxacin was initiated empirically because a ground-glass opacity with diffuse infiltration on the right lower lung was detected on chest radiograph. As we were not suspicious of phayngitis in such a patient with high grade fever under ciprofloxacin therapy, we did not feel the necessity of a pharynx culture. Splenomegaly and increased bilateral renal parenchymal echogenicity were seen on abdominal ultrasonography. On the second day of admission another two blood cultures were drawn. BD BACTEC ${ }^{\text {TM }} 9240$ blood culture system detected positivities of the first two blood cultures on the second day of admission. Gram staining revealed gram positive cocci and vancomycin was begun. Transthoracic echocardiogram (TTE) revealed no vegetation on any of the heart valves. Mild mitral and tricuspid regurgitation, moderate aortic regurgitation were noticed on TTE. Abdominal tomography revealed paraaortic, paracaval and mesenteric pathologic lymphadenopathies, a splenic infarct and a solid mass resembling renal cell carcinoma on the left kidney. Levofloxacin and vancomycin were stopped on the $3^{\text {rd }}$ day of admission as $S$. pyogenes were identified from the first two blood cultures and the second two blood cultures gave a positivity signal. The $\beta$-hemolytic colonies on blood agar plates were catalase negative and pyrrolidonyl peptidase enzyme activity positive. The presumptive identification of S. pyogenes was confirmed by bacitracin sensitivity and sulfamethoxazole-trimethoprim resistance. A latex agglutination test (SLIDEX STREPTO PLUS, France, Biomérieux) was used for the identification of Lancefield group antigen. Penicillin G at a total daily intravenous dose of 24 million units was started. Fever subsided on the $5^{\text {th }}$ day of hospitalization. Finally four blood culture bottles which were drawn on the first and second day of admission revealed $S$. pyogenes and two sets of blood cultures which were taken on the $6^{\text {th }}$ and $19^{\text {th }}$ day of hospitalization were negative, respectively.

Transesophageal echocardiogram (TEE) was performed on the $6^{\text {th }}$ day of hospitalization. There was mild to moderate mitral regurgitation and a mobile filamentous structure of 0.6 $\mathrm{cm}$ length attached to the anterior mitral leaflet which was suggestive of a small vegetation (Figure 1). TEE also yielded moderate to severe eccentric aortic regurgitation, however no vegetation was detected (Figure 2). The Departments of Cardiology and Cardiovascular Surgery agreed not to oper- 


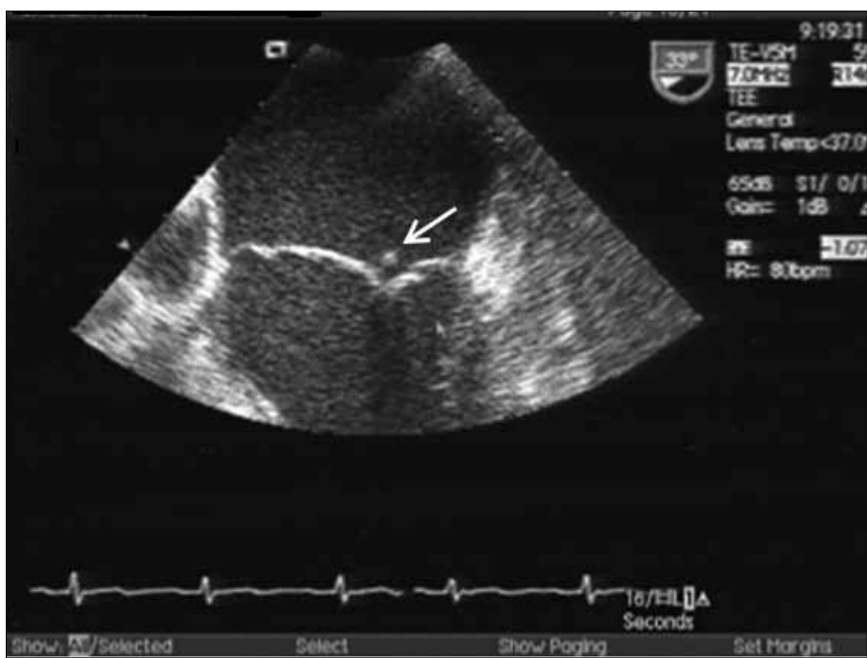

Figure 1. A small vegetation attached to mitral anterior leaflet was demonstrated with TEE

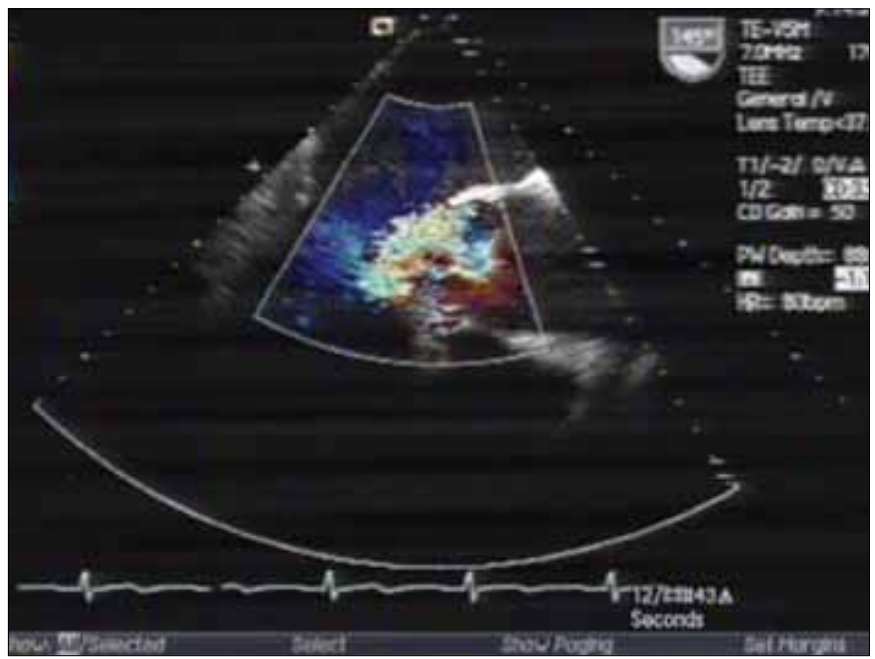

Figure 2. Moderate to severe aortic regurgitation with color Doppler echocardiography

ate on the patient on an urgent basis and to follow with periodic echocardiographic evaluations during antibiotic treatment. The Department of Urology also postponed operation until treatment of IE was completed. The second TEE which was done on the $14^{\text {th }}$ day of treatment showed constancy of the mitral valve vegetation with no major change in size and nodular appearance on the left coronary cusp of the aortic valve suggestive of a new vegetation (Figure 3). Selective coronary angiography done before valve replacement yielded coronary artery disease. Intraoperative macroscopic inspection revealed a fine nonverrucous filamentous structure on the anterior mitral leaflet and there was no chordal rupture. Mitral and aortic valves were replaced with a $27 \mathrm{~mm}$ and a 25 $\mathrm{mm}$ St. Jude mechanical prosthesis respectively on the $22^{\text {nd }}$ day of treatment. Excised valve tissues were not examined in the pathology laboratory therefore evidence of endocarditis could not be found microscopically. No growth was seen on the cultures of both valve tissues. Penicillin $G$ treatment was continued for a 6-week course. He was discharged from hos-

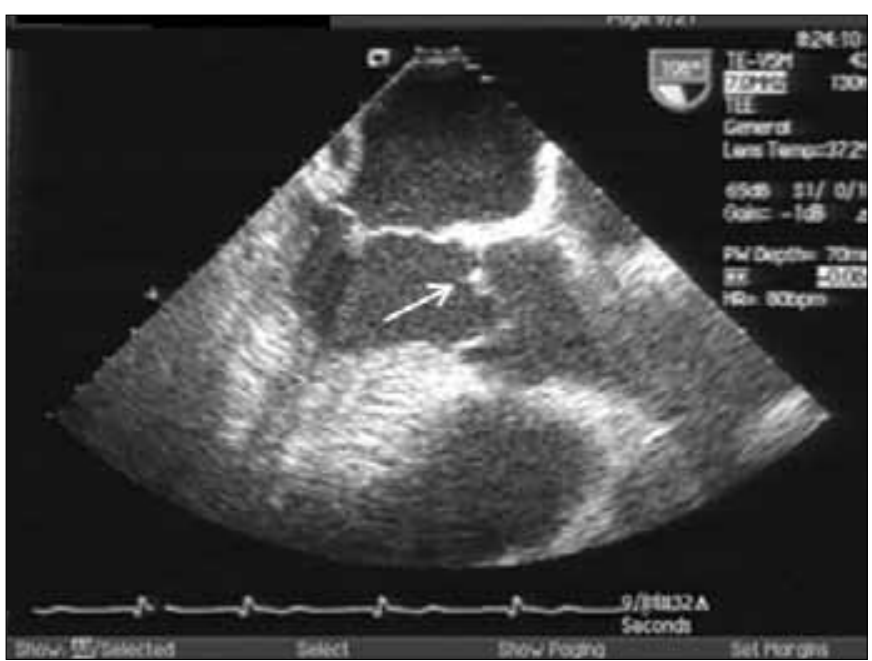

Figure 3. Nodular thickening of the aortic valve suggesting new vegetation

pital to be followed by the Urology Department for his solid organ malignancy.

\section{Discussion}

S. pyogenes is the most frequent bacterial cause of acute phayngitis and it also gives rise to a variety of cutaneous and systemic infections. IE due to $S$. pyogenes is uncommon in any age group and the onset is almost always acute. It was reported mostly following upper respiratory tract and skin infections in all age groups, but preceeding varicella infection in children and intravenous drug addiction in adults were the other predisposing factors (2-9). In our case, the source of bacteremia was not found. There is a tendency to involve normal left-sided valves as in our case $(2,6-8,10)$. S. pyogenes endocarditis in intravenous drug addicts, similar to Staphylococcus aureus, involves mainly right-sided valves and mortality is low (2).

In the presented case the definite diagnosis of IE was based on two major criteria preoperatively, and no risk factor for endocarditis was identified (1). The missing point in this case is the absence of tissue confirmation. However, the patient was treated in accordance with the guideline when the clinical, laboratory and TEE features were compatible with IE (1).

In the literature, malignancy was found to be an underlying medical condition in patients with endocarditis due to $\beta$-hemolytic streptococci $(4,11)$. Our case was interesting with the co-diagnosis of solid organ malignancy and IE caused by an infrequent bacteriologic agent during the evaluation of fever.

High dose penicillin $\mathrm{G}$ with or without gentamisin was the treatment of choice $(9,11)$. Although penicillin was the treatment of choice, valve replacement surgery was necessary in this case. New vegetation that was seen in another valve even under appropriate medical therapy was an indication for open heart surgery in accordance with Baddour's study (11). Vegetation, abscess and new dehiscence of the prosthetic valve are major TEE findings of IE. However vegetations less than $2 \mathrm{~mm}$ 
may not be detected with TEE. TEE should be repeated when there is significant valvular regurgitation with clinical suspicion of IE as in our case.

\section{Conclusion}

By presenting this patient, we emphasize that a rare cause of endocarditis, S. pyogenes, can be seen in a patient without any known risk factors. Blood cultures taken before prescribing antibiotics is essential in the diagnosis of any infection in a feverish patient. Valve replacement surgery in addition to antibiotic therapy is generally indicated for the IE cases caused by $S$. pyogenes.

\section{Conflict of Interest}

No conflict of interest was declared by the authors.

\section{References}

1. Habib G, Hoen B, Tornos P, Thuny F, Prendergast B, Vilacosta I, et al. Guidelines on the prevention, diagnosis, and treatment of infective endocarditis (new version 2009): the Task Force on the Prevention, Diagnosis, and Treatment of Infective Endocarditis of the European Society of Cardiology (ESC). Endorsed by the European Society of Clinical Microbiology and Infectious Diseases (ESCMID) and the International Society of Chemotherapy (ISC) for Infection and Cancer. Eur Heart J 2009;30:2369-413. [CrossRef]
2. Burkert T, Watanakunakorn C. Group A streptococcus endocarditis: report of five cases and review of literature. J Infect 1991;23:307-16. [CrossRef]

3. Branch J, Suganami Y, Kitagawa I, Stein GH, Tanaka E. A rare case of Group A Streptococcal endocarditis with absence of valvular vegetation. Inter Med 2010;49:1657-61. [CrossRef]

4. Lefort A, Lortholary O, Casassus P, Selton-Suty C, Guillevin L, Mainardi JL. Comparison between adult endocarditis due to $\beta$-hemolytic Streptococci (serogroups A, B, C, and G) and Streptococcus milleri. Arch Intern Med 2002;162:2450-6. [CrossRef]

5. Roca B, Marco JM. Presentation and outcome of infective endocarditis in Spain: a retrospective study. Int J Infect Dis 2007;11:198-203. [CrossRef]

6. Winterbotham A, Riley S, Kavanaugh-McHugh A, Dermody TS. Endocarditis caused by group A beta-hemolytic Streptococcus in an infant: case report and review. Clin Infect Dis 1999;29:196-8. [CrossRef]

7. Mohan UR, Walters S, Kroll JS. Endocarditis due to group A $\beta$-hemolytic Streptococcus in children with potentially lethal sequelae: 2 cases and review. Clin Infect Dis 2000;30:624-5. [CrossRef]

8. Benes J, Picha D, Kabelkova M, Dzupova O, Horova B, Gabrielova A. Infective endocarditis caused by unusual gram-positive pathogens. Folia Microbiol 2002;47:737-41. [CrossRef]

9. Laskey AL, Johnson TR, Dagartzikas MI, Tobias JD. Endocarditis attributable to group $A \beta$-hemolytic streptococcus after uncomplicated varicella in a vaccinated child. Pediatrics 2000;106:E40. [CrossRef]

10. Ozdemir N, Kaymaz C, Ozkan M. An unusual image within the left atrium with UFO-like appearance on transoesophageal echocardiography. Heart 2002;88:633. [CrossRef]

11. Baddour LM. Infective endocarditis caused by beta-hemolytic streptococci. The Infectious Diseases Society of America's Emerging Infections Network. Clin Infect Dis 1998;26:66-71. [CrossRef] 